GLASNIK MATEMATIČKI

Vol. 39(59)(2004), $265-272$

\title{
ON THE LINEAR COMBINATION OF THE REPRESENTATIONS OF STARLIKENESS AND CONVEXITY
}

\author{
Nikola Tuneski And Roza Aceska
}

Ss. Cyril and Methodius University Skopje, Macedonia

\begin{abstract}
Let $\mathcal{A}$ be the class of analytic functions in the unit disk $\mathcal{U}=\{z:|z|<1\}$ that are normalized with $f(0)=f^{\prime}(0)-1=0$. Also, let $S^{*}[A, B],-1 \leq B<A \leq 1$, be the class of functions $f \in \mathcal{A}$, such that $\frac{z f^{\prime}(z)}{f(z)} \prec \frac{1+A z}{1+B z}$, where "ঝ" denotes the usual subordination. In this paper we investigate the linear combination of the analytic representations of starlikeness and convexity and give sharp sufficient conditions over the differential operator

$$
a \frac{z f^{\prime}(z)}{f(z)}+b\left(1+\frac{z f^{\prime \prime}(z)}{f^{\prime}(z)}\right)
$$

that imply $f \in S^{*}[A, B]$. In that purpose we use the method of differential subordinations. Several corollaries and examples for different choices of $A$, $B, a$ and $b$ are given and comparison with previous known results is done.
\end{abstract}

\section{INTRODUCTION AND PRELIMINARIES}

Let $\mathcal{A}$ denote the class of functions $f(z)$ that are analytic in the unit $\operatorname{disk} \mathcal{U}=\{z:|z|<1\}$ and normalized by $f(0)=f^{\prime}(0)-1=0$. Further, let $f, g \in \mathcal{A}$. Then we say that $f(z)$ is subordinate to $g(z)$, and we write $f(z) \prec g(z)$, if there exists a function $\omega(z)$, analytic in the unit disk $\mathcal{U}$, such that $\omega(0)=0,|\omega(z)|<1$ and $f(z)=g(\omega(z))$ for all $z \in \mathcal{U}$. Specially, if $g(z)$ is univalent in $\mathcal{U}$ than $f(z) \prec g(z)$ if and only if $f(0)=g(0)$ and $f(\mathcal{U}) \subseteq g(\mathcal{U})$.

2000 Mathematics Subject Classification. 30C45.

Key words and phrases. Starlike function, starlike function of order $\alpha$, criteria, differential subordination, Jack lemma. 
A function $f \in \mathcal{A}$ belongs to the class $S^{*}[A, B]$, where $-1 \leq B<A \leq 1$, if and only if

$$
\frac{z f^{\prime}(z)}{f(z)} \prec \frac{1+A z}{1+B z}
$$

Geometrically, this means that the image of $\mathcal{U}$ by $z f^{\prime}(z) / f(z)$ is inside the open disk centered on the real axis with diameter end points $(1-A) /(1-B)$ and $(1+A) /(1+B)$. Special selection of $A$ and $B$ lead us to the following classes:

- the class of starlike functions of order $\alpha, 0 \leq \alpha<1: S^{*}(\alpha) \equiv S^{*}[1-$ $2 \alpha,-1]$;

- the class of starlike functions: $S^{*} \equiv S^{*}[1,-1]=S^{*}(0)$, with analytic representation $\operatorname{Re} z f^{\prime}(z) / f(z)>0, z \in \mathcal{U}$;

- the class of convex functions: $f(z)$ is convex if and only if $z f^{\prime}(z)$ is starlike, i.e., $\operatorname{Re}\left\{1+z f^{\prime \prime}(z) / f^{\prime}(z)\right\}>0, z \in \mathcal{U}$; and

- $S^{*}[\alpha, 0], 0<\alpha \leq 1$ : a class defined by $\left|z f^{\prime}(z) / f(z)-1\right|<\alpha, z \in \mathcal{U}$.

One can note that $S^{*}[A, B] \subseteq S^{*}((1-A) /(1-B))$.

The quotient of the analytic representations of convex and starlike functions, i.e., the expression $\frac{1+z f^{\prime \prime}(z) / f^{\prime}(z)}{z f^{\prime}(z) / f(z)}$, was studied in [7] and [6] as a criteria for starlikeness and starlikeness of order $\alpha$. These results were generalized in [8], where the same quotient was studied as a criteria for a function $f(z) \in \mathcal{A}$ to belong to the class $S^{*}[A, B]$. In this paper we investigate the linear combination of the analytic representations of starlikeness and convexity, i.e., the differential operator

$$
G(a, b, f ; z) \equiv a \frac{z f^{\prime}(z)}{f(z)}+b\left(1+\frac{z f^{\prime \prime}(z)}{f^{\prime}(z)}\right),
$$

and give sharp sufficient conditions that imply $f \in S^{*}[A, B]$. The criteria obtained for different choices of $A, B, a$ and $b$ are compared with previous known results. Implication

$$
G(1-\alpha, \alpha, f ; z) \in S^{*}[A, B] \quad \Rightarrow \quad \frac{z f^{\prime}(z)}{f(z)} \prec q(z)
$$

is already studied in [1], page 118, giving necessary conditions over $q(z)$. Therefore this work closes that subject.

For obtaining the main result we will use the method of differential subordinations. The general theory of differential subordinations, as well as the theory of first-order differential subordinations, was introduced by Miller and Mocanu in [2] and [3]. Namely, if $\phi: \mathbb{C}^{2} \rightarrow \mathbb{C}$ (where $\mathbb{C}$ is the complex plane) is analytic in a domain $D$, if $h(z)$ is univalent in $\mathcal{U}$, and if $p(z)$ is analytic in $\mathcal{U}$ with $\left(p(z), z p^{\prime}(z)\right) \in D$ when $z \in \mathcal{U}$, then $p(z)$ is said to satisfy a first-order differential subordination if

$$
\phi\left(p(z), z p^{\prime}(z)\right) \prec h(z) .
$$


The univalent function $q(z)$ is said to be a dominant of the differential subordination (1) if $p(z) \prec q(z)$ for all $p(z)$ satisfying (1). If $\widetilde{q}(z)$ is a dominant of (1) and $\widetilde{q}(z) \prec q(z)$ for all dominants of (1), then we say that $\widetilde{q}(z)$ is the best dominant of the differential subordination (1).

From the theory of first-order differential subordinations we will make use of the following lemma.

Lemma $1.1([3])$. Let $q(z)$ be univalent in the unit disk $\mathcal{U}$, and let $\theta(\omega)$ and $\phi(\omega)$ be analytic in a domain D containing $q(\mathcal{U})$, with $\phi(\omega) \neq 0$ when $\omega \in q(\mathcal{U})$. Set $Q(z)=z q^{\prime}(z) \phi(q(z)), h(z)=\theta(q(z))+Q(z)$, and suppose that

i) $Q(z)$ is starlike in the unit disk $\mathcal{U}$; and

ii) $\operatorname{Re} \frac{z h^{\prime}(z)}{Q(z)}=\operatorname{Re}\left\{\frac{\theta^{\prime}(q(z))}{\phi(q(z))}+\frac{z Q^{\prime}(z)}{Q(z)}\right\}>0, z \in \mathcal{U}$.

If $p(z)$ is analytic in $\mathcal{U}$, with $p(0)=q(0), p(\mathcal{U}) \subseteq D$ and

$$
\theta(p(z))+z p^{\prime}(z) \phi(p(z)) \prec \theta(q(z))+z q^{\prime}(z) \phi(q(z))=h(z)
$$

then $p(z) \prec q(z)$, and $q(z)$ is the best dominant of (2).

\section{Main Results And CONSEQUences}

In the beginning, using Lemma 1.1 we will prove the following useful result.

Lemma 2.1. Let $p(z)$ be analytic in the unit disk $\mathcal{U}, p(0)=1,0 \notin p(\mathcal{U})$ and let $a, b, A$ and $B$ be real numbers such that $-1 \leq B<A \leq 1$ and $1+\frac{a}{b}+\frac{1-A B}{(1+|A|)^{2}} \geq 0$. If

$$
(a+b) p(z)+b \frac{z p^{\prime}(z)}{p(z)} \prec(a+b) \frac{1+A z}{1+B z}+\frac{b(A-B) z}{(1+A z)(1+B z)} \equiv h_{1}(z)
$$

then $p(z) \prec \frac{1+A z}{1+B z} \equiv q(z)$. Function $q(z)$ is the best dominant of (3).

Proof. We choose $\theta(\omega)=(a+b) \omega$ and $\phi(\omega)=b / \omega$. Then $q(z)$ is univalent in $\mathcal{U} ; \theta(\omega)$ and $\phi(\omega)$ are analytic with domain $D=\mathbb{C} \backslash\{0\}$ which contains $q(\mathcal{U})$ and $\phi(\omega) \neq 0$ when $\omega \in q(\mathcal{U})$. Further,

$$
Q(z)=z q^{\prime}(z) \phi(q(z))=\frac{b(A-B) z}{(1+A z)(1+B z)}
$$

is starlike because for $z=e^{i \lambda}, \lambda \in[-\pi, \pi]$, we have

$\operatorname{Re} \frac{z Q^{\prime}(z)}{Q(z)}=\operatorname{Re} \frac{1-A B z^{2}}{(1+A z)(1+B z)}=\frac{(1-A B)[1+A B+(A+B) \cos \lambda]}{\left|1+A e^{i \lambda}\right|^{2}\left|1+B e^{i \lambda}\right|^{2}} \geq 0$.

Next, let $h(z)=\theta(q(z))+Q(z)$ and $z=e^{i \lambda}, \lambda \in[-\pi, \pi]$. Then

$$
\operatorname{Re} \frac{z h^{\prime}(z)}{Q(z)}=\frac{1+A B+(A+B) \cos \lambda}{\left|1+B e^{i \lambda}\right|^{2}} \cdot\left(1+\frac{a}{b}+\frac{1-A B}{\left|1+A e^{i \lambda}\right|^{2}}\right) \geq 0 .
$$


Also, $h(z)=h_{1}(z), p(0)=q(0)=1$ and $p(\mathcal{U}) \subseteq D$. Therefore, the conditions of Lemma 1.1 are satisfied and concerning that subordinations (2) and (3) are equivalent we receive the conclusion of Lemma 2.1.

Remark 2.2. For $a=1-\alpha, b=\alpha, A=1$ and $B=-1$ in Lemma 2.1 we have an improvement of a result published in [1], page 251. The improvement is made over the condition for $\alpha$. Namely, here we have condition $\alpha \notin(-2,0)$ and in [1] there is $\alpha>0$.

Putting $p(z)=\frac{z f^{\prime}(z)}{f(z)}$ in Lemma 2.1 we obtain a criteria for a function $f(z)$ to belong to the class $S^{*}[A, B]$.

TheOrem 2.3. Let $f \in \mathcal{A}$ and let $a, b, A$ and $B$ be real numbers such that $-1 \leq B<A \leq 1$ and $1+\frac{a}{b}+\frac{1-A B}{(1+|A|)^{2}} \geq 0$. If

$$
G(a, b, f ; z) \prec h_{1}(z)
$$

then $f \in S^{*}[A, B]$. Function $\frac{1+A z}{1+B z}$ is the best dominant of (4).

Theorem 2.3 can be rewritten in the following, equivalent form. If

Theorem 2.4. Let $f \in \mathcal{A}$ and let $A$ and $B$ be such that $-1 \leq B<A \leq 1$.

or

$$
G(1,-1, f ; z) \prec \frac{(B-A) z}{(1+A z)(1+B z)} \equiv h_{2}(z)
$$

$$
\begin{gathered}
G(1-\alpha, \alpha, f ; z) \prec \frac{1+A z}{1+B z}+\alpha \frac{(A-B) z}{(1+A z)(1+B z)} \equiv h_{3}(z), \\
\alpha \notin\left(-\frac{(1+|A|)^{2}}{1-A B}, 0\right),
\end{gathered}
$$

then $f \in S^{*}[A, B]$. Function $\frac{1+A z}{1+B z}$ is the best dominant of (5) and (6).

Proof. Equivalency of Theorem 2.3 and Theorem 2.4 follows from the fact that either $a+b=0$ or $a+b \neq 0$. In the first case, after dividing both sides of subordination (4) with $a$ we receive subordination (5). Similarly, in the second case, $a+b \neq 0$, after dividing both sides of subordination (4) with $a+b$ and putting $\alpha=\frac{b}{a+b}$ we obtain subordination (6).

Choosing appropriate values of $A$ and $B$ in Theorem 2.4 we receive the following examples.

EXAMPLE 2.5. Let $f \in \mathcal{A}$.

i) If $G(1,-1, f ; z) \prec-\frac{2 z}{1-z^{2}}$ then $f \in S^{*}$. $(A=1$ and $B=-1$ in Theorem $2.4)$

ii) If $0 \leq \beta<1$ and $G(1,-1, f ; z) \prec-\frac{2(1-\beta) z}{(1-z)[1+(1-2 \beta) z]}$ then $f \in S^{*}(\beta)$. $(A=1-2 \beta$ and $B=-1$ in Theorem 2.4$)$; 
iii) If $0<\gamma \leq 1$ and $G(1,-1, f ; z) \prec-\frac{\gamma z}{1+\gamma z}$ then $\left|z f^{\prime}(z) / f(z)-1\right|<\gamma$, $z \in \mathcal{U}$. $(A=\gamma$ and $B=-1$ in Theorem 2.4);

iv) If $\alpha \notin(-2,0)$ and $G(1-\alpha, \alpha, f ; z) \prec \frac{1+z}{1-z}+\frac{2 \alpha z}{1-z^{2}}$ then $f \in S^{*} .(A=1$ and $B=-1$ in Theorem 2.4);

v) If $0 \leq \beta<1, \alpha \notin\left(-\frac{[1+|1-2 \beta|]^{2}}{2(1-\beta)}, 0\right)$ and $G(1-\alpha, \alpha, f ; z) \prec \frac{1+(1-2 \beta) z}{1-z}+$ $\frac{2 \alpha(1-\beta) z}{(1-z)[1+(1-2 \beta) z]}$ then $f \in S^{*}(\beta) .(A=1-2 \beta$ and $B=-1$ in Theorem $2.4) ;$ and

vi) If $0<\gamma \leq 1, \alpha \notin\left(-(1+\gamma)^{2}, 0\right)$, and $G(1-\alpha, \alpha, f ; z) \prec 1+\gamma z+\frac{\alpha \gamma z}{1+\gamma z}$ then $\left|z f^{\prime}(z) / f(z)-1\right|<\gamma, z \in \mathcal{U}$. $(A=\gamma$ and $B=0$ in Theorem 2.4).

REMARK 2.6. Results (v) and (vi), $\gamma=1$, from Example 2.5 improve the results from [4] and [5], respectively, since the last ones are stated only for $\alpha>0$.

Next, using Theorem 2.4, we study the modulus of $G(a, b, f ; z)$ as a sufficient condition that implies $f \in S^{*}[A, B]$.

Corollary 2.7. Let $f \in \mathcal{A}$ and let $A$ and $B$ be such that $-1 \leq B<A \leq$ 1. If

$$
|G(1,-1, f ; z)|<\frac{A-B}{(1+|A|)(1+|B|)} \equiv \delta_{1}
$$

then $f \in S^{*}[A, B]$. This result is sharp.

Proof. From $\min \left\{\left|h_{2}\left(e^{i \lambda}\right)\right|: \lambda \in[-\pi, \pi]\right\}=\delta_{1}$ we obtain that inequality (7) implies subordination (5) and further $f \in S^{*}[A, B]$. The result is sharp as the function

$$
f(z)=\left\{\begin{array}{cc}
z(1+B z)^{(A-B) / B}, & B \neq 0 \\
f(z)=z e^{A z}, & B=0
\end{array} .\right.
$$

shows, since $f \in S^{*}[A, B]$ and $\left|G\left(1,-1, f ; e^{i \lambda}\right)\right|=\delta_{1}$, for appropriate choice of $\lambda \in\{0, \pi\}$.

Corollary 2.8. Let $f \in \mathcal{A}, \alpha \geq 0$ and let $A$ and $B$ be such that $-1 \leq$ $B<A \leq 1$. Also, let us define two sets of conditions:

(a) $A B<0,4 A B<(A+B)(1+A B) \leq 4 A|B|, \alpha>\alpha_{1}^{*}$;

(b) $A B<0,(A+B)(1+A B)>4 A|B|, \alpha_{1}^{*}<\alpha<\alpha_{2}^{*}$.

Here $\alpha_{1}^{*}$ and $\alpha_{2}^{*}$ are the bigger real roots of the equations $\eta(A, B ; \alpha) \equiv a_{1} \alpha^{2}+$ $b_{1} \alpha+c_{1}=0$ and $\eta(-A,-B ; \alpha)=0$, respectively, where $a_{1}=A(1-B)^{2}+$ $B(1-A)^{2}, b_{1}=(1-A)\left[A\left(1-B^{2}\right)(1+A)+2 B(1-A)^{2}\right]$ and $c_{1}=B(1-A)^{4}$. Further, let

$$
\delta_{2}=\left\{\begin{array}{cc}
\sqrt{\frac{A(1+\alpha)}{(A+B)(1+A B)+4 A B t_{*}}}, & \text { if }(a) \text { or }(b) \text { holds }, \\
\min \left\{\frac{1+\alpha-A}{(1-A)(1-B)}, \frac{1+\alpha+A}{(1+A)(1+B)}\right\}, & \text { otherwise, }
\end{array}\right.
$$


where

$$
t_{*}=-\frac{A}{2(1+\alpha)}-\frac{1+\alpha}{2 A}-\frac{\sqrt{\alpha B\left(1+\alpha-A^{2}\right)(1+\alpha-A B)(B(1+\alpha)-A)}}{2 A B(1+\alpha)}
$$

Under this notations, if

$$
|G(1-\alpha, \alpha, f ; z)-1| \leq(A-B) \delta_{2}
$$

then $f \in S^{*}[A, B]$. This result is sharp.

Proof. In order to prove $f \in S^{*}[A, B]$ it is enough to show that inequality (9) implies subordination (6), i.e., to show that $\min \left\{\left|h_{3}\left(e^{i \lambda}\right)-1\right|: \lambda \in\right.$ $[-\pi, \pi]\}=(A-B) \delta_{2}$. Thus, we will study the minimum of the function $\Psi(t)$ defined by

$$
\left|h_{3}\left(e^{i \lambda}\right)-1\right|^{2}=(A-B)^{2} \frac{(1+\alpha)^{2}+A^{2}+2 A t(1+\alpha)}{\left(1+2 A t+A^{2}\right)\left(1+2 B t+B^{2}\right)}=(A-B)^{2} \cdot \Psi(t),
$$

$t=\cos \lambda \in[-1,1]$, and show that $\Delta=\min \{\Psi(t): t \in[-1,1]\}=\delta_{2}^{2}$.

First we note that $\Psi(t)$ is continues function at any $t \in(-1,1)$ and the equation $\Psi^{\prime}(t)=0$ has only two solutions $t_{1,2}=\varphi(A, \alpha) \pm \frac{\sqrt{D}}{2 A B(1+\alpha)}$, where $\varphi(A, \alpha)=-\frac{A}{2(1+\alpha)}-\frac{1+\alpha}{2 A}$ and $D=\alpha B\left(1+\alpha-A^{2}\right)(1+\alpha-A B)(B(1+$ $\alpha)-A)$. Even more, if both solutions are real then at least one of them lies outside the interval $(-1,1)$ because of $|\varphi(A, \alpha)|=1+\frac{(1+\alpha-|A|)^{2}}{2|A|(1+\alpha)}$. So, $\Psi(t)$ is ether monotonous function, or has only one local extremal point on $(-1,1)$. Consequently, $\Psi(t)$ attains its minimal value at some interior point $t_{*} \in(-1,1)$ if and only if $\Psi(t)$ has local minimum on $(-1,1)$, i.e., if and only if

$$
\Psi^{\prime}(-1)=-\frac{2 \cdot \eta(A, B ; \alpha)}{(1-A)^{4}(1-B)^{4}}<0 \quad \text { and } \quad \Psi^{\prime}(1)=\frac{2 \cdot \eta(-A,-B ; \alpha)}{(1+A)^{4}(1+B)^{4}}>0,
$$

or equivalently

$$
\eta(A, B ; \alpha)>0 \quad \text { and } \quad \eta(-A,-B ; \alpha)>0 .
$$

It remains to show that inequalities (10) hold if and only if one of the disjoint sets of condition (a) or (b) is satisfied.

If we rewrite $\eta(A, B ; \alpha)$ as

$$
\eta(A, B ; \alpha)=A \alpha(1-B)^{2}\left(1+\alpha-A^{2}\right)+B(1-A)^{2}(1+\alpha-A)^{2}
$$

than it is easy to verify that necessary condition for the inequalities (10) is $-1 \leq B<0<A \leq 1$, i.e., $A B<0$.

Next, let $A B<0$. Then

$$
a_{1}-b_{1}=\left[2-(1-B)^{2}\right] A^{3}-B\left[5 A^{2}-2(1+B) A+1\right]>0
$$

and $c_{1} \leq 0$. Thus, simple analysis of the quadratic function $\eta(A, B ; \alpha)$ shows that when $\alpha \geq 0$,

$$
\eta(A, B ; \alpha)>0 \quad \Leftrightarrow \quad a_{1}>0 \text { and } \alpha>\alpha_{1}^{*},
$$


where $a_{1}>0 \Leftrightarrow 4 A B<(A+B)(1+A B)$ and $\alpha_{1}^{*}$ is the bigger real root of the equation $\eta(A, B ; \alpha)=0$ solved for $\alpha$, while the smaller root is negative. Indeed, if $b_{1}<a_{1}<0$ then $\eta(A, B ; \alpha)<0$ for any $\alpha \geq 0$, because $\eta(A, B ; 0)=c_{1} \leq 0$ and because the parabola $\eta(A, B ; \alpha)$ is concave upward with zeros $\alpha_{1}$ and $\alpha_{2}$ such that $\alpha_{1} \alpha_{2}=\frac{c_{1}}{a_{1}} \geq 0$ and $\alpha_{1}+\alpha_{2}=-\frac{b_{1}}{a_{1}}<0$, i.e., $\alpha_{1}, \alpha_{2}<0$.

Similarly, if $A B<0$, for the quadratic equation $\eta(-A,-B ; \alpha)=a_{2} \alpha^{2}+$ $b_{2} \alpha+c_{2}$ with $a_{2}=-A(1+B)^{2}-B(1+A)^{2}, b_{2}=(1+A)\left[-A\left(1-B^{2}\right)(1-\right.$ $\left.A)-2 B(1+A)^{2}\right]$ and $c_{2}=-B(1+A)^{4}$, we have $\eta(A, B ; 0)=c_{2}>0$ and $b_{2}-a_{2}=\left[2-(1+B)^{2}\right] A^{3}-5 B A^{2}-2 B(1-B) A-B>0$. Therefore, when $\alpha \geq 0$, inequality $\eta(-A,-B ; \alpha)=a_{2} \alpha^{2}+b_{2} \alpha+c_{2}>0$ has two solutions. First one

$$
\alpha \geq 0 \quad \text { when } \quad a_{2} \geq 0,
$$

since $b_{2}>a_{2} \geq 0$ implies that the parabola $\eta(-A,-B ; \alpha)$ is concave downward with vertex on the left side of the $y$-axis, and the second one

$$
\alpha<\alpha_{2}^{*} \text { when } a_{2}<0,
$$

where $\alpha_{2}^{*}$ is the bigger real root of the equation $\eta(-A,-B ; \alpha)=0$, true because $a_{2}<0$ implies that the parabola $\eta(-A,-B ; \alpha)$ is concave upward with one positive and one negative zero, due to $\eta(-A,-B ; 0)>0$. Here $a_{2} \geq 0$ if and only if $(A+B)(1+A B) \leq 4 A|B|$.

Finally, conditions (11), (12) and (11), (13) can be sublimated into the conditions (a) and (b), respectively. The second is possible because $\eta\left(-A,-B ; \alpha_{1}^{*}\right)=-8 A B\left[A^{2}+\left(1+\alpha_{1}^{*}\right)^{2}\right]>0$ implies $\alpha_{1}^{*}<\alpha_{2}^{*}$.

From the above analysis we can say that inequalities (10) hold if and only if (a) or (b) is satisfied.

The sharpness of the result can be verified using function $f(z)$ defined by (8) such that $f \in S^{*}[A, B]$ and $\left|G\left(1-\alpha, \alpha, f ; e^{i \lambda}\right)-1\right|=\delta_{2}$ for appropriate choice of $\lambda, \cos \lambda \in\left\{-1, t_{*}, 1\right\}$.

If $A B \geq 0$ the corollary is much simpler.

Corollary 2.9. Let $f \in \mathcal{A}, \alpha \geq 0$ and let $A$ and $B$ be such that $-1 \leq$ $B<A \leq 1$ and $A B \geq 0$. If

$$
|G(1-\alpha, \alpha, f ; z)-1| \leq(A-B) \frac{1+\alpha+|A|}{(1+|A|)(1+|B|)}
$$

then $f \in S^{*}[A, B]$. This result is sharp.

The following example exhibits some concrete conclusions that can be obtained from the previous corollaries by specifying the values $A$ and $B$.

ExAmple 2.10. Let $f \in \mathcal{A}$ and $\alpha \geq 0$. The following results are sharp.

i) If $|G(1,-1, f ; z)|<1 / 2, z \in \mathcal{U}$, then $f \in S^{*}$. $(A=1$ and $B=-1$ in Corollary 2.7); 
ii) If $0 \leq \beta \leq 1 / 2$ then $|G(1,-1, f ; z)|<1 / 2, z \in \mathcal{U}$, implies $f \in S^{*}(\beta)$. $(A=1-2 \beta$ and $B=-1$ in Corollary 2.7$)$;

iii) If $1 / 2 \leq \beta<1$ then $|G(1,-1, f ; z)|<\frac{1-\beta}{2 \beta}, z \in \mathcal{U}$, implies $f \in S^{*}(\beta)$. $(A=1-2 \beta$ and $B=-1$ in Corollary 2.7$)$;

iv) If $0<\gamma \leq 1$ then $|G(1,-1, f ; z)|<1-\frac{1}{1+\gamma}, z \in \mathcal{U}$, implies $\left|\frac{z f^{\prime}(z)}{f(z)}-1\right|<\gamma, z \in \mathcal{U} .(A=\gamma$ and $B=0$ in Corollary 2.7);

v) $|G(1-\alpha, \alpha, f ; z)-1| \leq 1+\alpha, z \in \mathcal{U}$, implies $f \in S^{*}$. $(A=1$ and $B=-1$ in Corollary 2.8$)$;

vi) If $1 / 2 \leq \beta<1$ then $|G(1-\alpha, \alpha, f ; z)-1| \leq(1-\beta)\left(1+\frac{\alpha}{2 \beta}\right), z \in \mathcal{U}$, implies $f \in S^{*}(\beta) .(A=1-2 \beta$ and $B=-1$ in Corollary 2.9);

vii) If $0<\gamma \leq 1$ then $|G(1-\alpha, \alpha, f ; z)-1| \leq \gamma\left(1+\frac{\alpha}{1+\gamma}\right), z \in \mathcal{U}$, implies $\left|\frac{z f^{\prime}(z)}{f(z)}-1\right|<\gamma, z \in \mathcal{U} .(A=\gamma$ and $B=0$ in Corollary 2.9);

REMARK 2.11. The results (v) and (vii), $\gamma=1$, of Example 2.10 can be found on pages 252 and 257 in [1], respectively.

\section{REFERENCES}

[1] S.S. Miller and P.T. Mocanu, Differential subordinations, Theory and Applications, Marcel Dekker, New York-Basel, 2000.

[2] S.S. Miller and P.T. Mocanu, Differential subordinations and univalent functions, Michigan Math. J. 28 (1981), 157-171.

[3] S.S. Miller and P.T. Mocanu, On some classes of first-order differential subordinations, Michigan Math. J. 32 (1985), 185-195.

[4] P.T. Mocanu, Alpha-convex integral operator and starlike functions of order beta, Babeş-Bolyai Univ. Fac. Math. and Phys. Res. Sem., Internat Seminar on Functional Equations, Approximation and Convexity, Preprint 6 (1989), 231-238.

[5] P.T. Mocanu, On certain subclasses of starlike functions, Studia Univ. Babeş-Bolyai Math 34 (1994), 3-9.

[6] M. Obradović and N. Tuneski, On the starlike criteria defined by Silverman, Zeszyty Nauk. Politech. Rzeszowskiej Mat. 181 (2000), 59-64.

[7] H. Silverman, Convex and starlike criteria, Internat. J. Math. \& Math. Sci. 22 (1999), $75-79$.

[8] N. Tuneski, On the quotient of the representations of convexity and starlikeness, Math. Nachr. 248-249 (2003), 200-203.

N. Tuneski

Faculty of Mechanical Engineering

Karpoš II b.b., 1000 Skopje

Republic of Macedonia

E-mail: nikolat@mf.ukim.edu.mk

R. Aceska

Faculty of Mechanical Engineering

Karpoš II b.b., 1000 Skopje

Republic of Macedonia

E-mail: aroza@mf.ukim.edu.mk 